\title{
Probability and Style in the Chorales of J. S. Bach
}

\author{
MATTHEW WOOLHOUSE[1] \\ McMaster University
}

\begin{abstract}
This paper discusses de Clercq's (2015) contribution to our understanding of the relationship between scale degree and cadence type within Bach chorales from the perspective of style and probability. De Clercq is applauded for the diligence of this research and for attempting to synthesize findings into a practical, working model of benefit to music-theory students and educators. A literal interpretation of a premise underpinning his model - that more common musical events are more indicative of a style-is, however, found to be inconsistent. A test is described in which university students enrolled in a second-level harmony class were presented with pairs of cadences. Cadences were manipulated in various ways, primarily to investigate whether the inclusion of certain figurations would result in a perfect-authentic cadence, the most ubiquitous cadence within Bach chorales, being considered less stylistic than a never-occurring cadence. This proved to be the case, demonstrating the importance of figuration over scale degree and cadence for the accomplishment of style. De Clercq's model is further discussed with respect to probabilistic models of music and in relation to proscriptive approaches to teaching harmony.
\end{abstract}

Submitted 2014 December 10; accepted 2015 June 17.

KEYWORDS: J. S. Bach, chorales, cadences, probability, style, figuration

THE notion, in the contemporary field of music psychology at least, that sensitivity to style depends upon a listener's ability to detect the probabilities of musical events can be traced to Emotion and Meaning in Music, Leonard Meyer's influential monograph in which emotion and meaning are conceived in terms of expectations, either realized, delayed or thwarted (Meyer, 1956). As Meyer wrote, "As soon as the unexpected, or for that matter the surprising, is experienced, the listener attempts to fit it into the general system of beliefs relevant to the style of the work" (p. 29). In the intervening decades following the publication of Meyer's book, the concept of music and our relationship to it as a distributional process has given rise to an array of experimental studies demonstrating the importance of relative frequencies and transition probabilities of tones and chords (Saffran, Johnson, Aslin, \& Newport, 1999; Creel, Newport \& Aslin, 2004; Pearce, Ruiz, Kapasi, Wiggins, \& Bhattacharya, 2010), frequently with respect to artificial musical grammars (Kuhn \& Dienes, 2005; Loui, Wu, Wessel, \& Knight, 2009; Loui, Wessel \& Kam, 2010). Similarly, computational approaches to modeling melodic-pitch expectations have been undertaken (Pearce \& Wiggins, 2004; 2006), incorporating, for example, unsupervised statistical learning of sequential structure in music (Dubnov, Assayag, Lartillot, \& Bejerano, 2003; Pearce et al., 2010). And, given the philosophical and information-theoretic origins of Meyer's original insights (see also Meyer, 1957), perhaps unsurprisingly, probabilistic approaches within the world of music-theory have become equally prevalent, dating at least to the 1940's (Piston, 1941/1978; McHose, 1947) and more recently in corpus studies such as De Clercq \& Temperley (2011), Rohrmeier \& Cross (2008), Conklin (2006), and Zanette (2006), to mention but a few.

In Trevor de Clercq's analysis (this issue, 2015), and subsequent modeling of the relationship between scale degrees and cadences in the chorales of J. S. Bach, a probabilistic approach is also present. In toto, de Clercq's pedagogically motivated focus (a hope is that students will be able to harmonize in a more Bachian style) is to associate certain scale degrees with various cadences. Through an analysis of 371 chorale harmonizations by Bach, reduced to 346 for reasons of duplication, the statistical association of phrase-ending three-note scale patterns (e.g. 6-6-5 or 3-2-1), taking into account local modulations, is 
calculated with respect to perfect authentic (PA), imperfect authentic (IA), half (HF), phrygian (PH), plagal (PL), and deceptive (DE) cadences. Reducing the phrase-ending scale pattern to a single note, the last, accounts for about $80 \%$ of the 30 most-common non-final cadential events in Bach chorales-final cadences were removed from the analysis due to their high predictability (166 of the 177 major-key chorales end with a PA in the tonic). Simplified tabular major- and minor-key models (de Clercq, 2015, Figs. 7 and 8), incorporating the main findings of the analysis and including modulations to closely related keys, show the most likely cadences given a phrase ending on any of the seven scale degrees. Finally, the model's accuracy is further improved by taking into account the melodic interval leading into the cadence. Perhaps unsurprisingly, given the etymological root of "cadence" (Ln. cadere; fall), for phrases terminating with a melodic descending second the success rate improves to $90 \%$.[2]

First, de Clercq should be applauded, not only for his painstaking, literally hand-crafted researchcadence categorization was done at the piano by ear-but also for his efforts to synthesize findings into a practical, working model of benefit to music-theory students and educators. A conscious effort has been made to distill empirical observation into "rules-of-thumb", whereby a seemingly huge array of possibilities-bewildering to many novice harmonizers - is ultimately reduced to a comprehensive flowchart (de Clercq, 2015, Figure 18), in which, given a phrase-ending two-note melodic pattern, a student can arrive at a cadence the Master himself would likely have chosen. For music-theory professors who might rely excessively on implicit harmonic knowledge rather than explicit didactic tools, as in truth many of us probably do, models of this sort will doubtless be a boon. Which is to say, the "but why?" question, which all too often is inadequately dealt with in the harmony classroom, can, at least in respect of appropriate cadences, be addressed with the answer: given $x$ and $y$, Bach does $z$-you should too! The drawbacks of potentially proscriptive approaches to teaching harmony are acknowledged by de Clercq, and are discussed below.

Second, from the perspective of empirical musicology, de Clercq's research adds to a growing body of knowledge demonstrating the functionally constrained nature of tonal-harmonic music, which in the case of Bach had in earlier decades only been surmised (e.g. Lester, 1999, p. vii). For example, in Bach chorales, the frequency of harmonic n-grams has been shown to be inversely proportional to their frequency rank, obeying a Zipf distribution - that is, the most frequent two-, three-, four- or even fivechord progression is roughly twice as frequent as the second most common progression (of identical length), three times as frequent as the third most common, and so on (Rohrmeier \& Cross, 2008). As Clercq's Tables 5 and 6 show, cadences that rank highly similarly dominate the distribution of all cadences in major- and minor-key chorales. Indeed, if scale degree is ignored, the top-three ranking cadences in Tables 5 and 6 (PA1, HF5, IA3) account for 1332 of the 1378 cadences explicitly listed, some 97\%.[3]

A particular distinction of the research, however, lies in the combination of melodic and harmonic content in the analysis, an approach lacking in some corpus studies where abstracted harmony alone has been considered. The advantage of this is twofold. First, pedagogically, students are usually given a chorale tune and asked to add lower parts, echoing the scenario in which Bach himself harmonized preexisting hymn melodies.[4] De Clercq's melody-centered model is therefore tailor-made to the requirements of typical undergraduate harmony classes. Second, this research highlights an interaction between melodic scale-degree, chord element (root, third, fifth, etc.) and chord function, and in so doing opens a number of lines of inquiry. For example, what might explain the prevalence of HFs terminating with the fifth of the chord in the melody rather than the third, or, for that matter, the prevalence of IAs terminating with a melodic third rather than fifth? Psychoacoustic models taking into account local voice leading, such as Parncutt's perceptual-roots model (Parncutt, 1997), may yield insight; a cognitive explanation incorporating the bottom-up component of Eugene Narmour's (1990) implication-realization model for melodic expectancy might also be predictive (e.g. Krumhansl, 1995). Either way (and I encourage de Clercq to explore these and other possibilities), the assumption put forward is that these patterns are integral to Bach's musical style - "With this information, we can better know when and to where ... to modulate at phrase endings if we want to simulate the Bach chorale style..." (de Clercq, 2015, p. 191). Surprisingly, given the copious support garnered in favor of the probability-style position since Meyer's influential book, this assumption is perhaps where de Clercq's thesis might begin to be interrogated.

That more common musical events are more indicative of a style seems to be self evident to the point of being unchallengeable, and I am not about to commit musicological heresy by questioning this general premise. After all, given the cadence-melody distributions de Clercq has uncovered, it is perfectly reasonable of him to state that if a melody ends on a particular scale-degree, "...it would be more stylistic to modulate [for example] to the dominant ... than to have a half cadence in tonic" (p. 188; my emphasis). 
However, extended logically this approach can appear a little absurd. Take, for example, the model as laid out in Tables 7 and 8. If we are to accept at face value the assumption that more common musical events are more symptomatic of a style, then we have to conclude that cadences to the right of the tables are less stylistic than those to the left, that rarely occurring cadences are somehow less Bachian. But is this really so? Is it the case, as in Table 7, that a HF in the supertonic, where the melody note is the fifth of the chord (i.e. third scale-degree), is somehow less indicative of Bach's chorale style than an equivalent cadence in the submediant (melody note, seventh scale degree), simply by virtue of its relatively infrequent occurrence? Similarly, in Table 8, an IA in the mediant, where the melody note is the third of the chord (i.e. fifth scale-degree), appears to be more stylistic than an equivalent cadence in the subdominant (melody note, sixth scale degree). Intuitively, at least, it seems highly unlikely that these frequency-of-occurrence differences lead to some cadences being perceived as more stylistic (Bachian, even) than others. However, the conclusion that this is indeed the case arguably falls out of the model, and in so doing might suggest that the proposition upon which it is based at least requires qualification. Which is to say, the relationship between style and probability in respect of musical events - here, a cadence in a particular key given a melodic pitch-might not be straightforward.

Inspired by de Clercq's research-manship, in the course of writing this review I endeavored to explore this matter, albeit in a very perfunctory fashion. My aim was to test whether students, versed in four-part harmony à la Bach chorales, could distinguish between more- and less-stylistic cadences based upon the frequencies reported by de Clercq. Following an initial test in which students in my second-level undergraduate harmony class exhibited zero stylistic preference for cadences belonging either to the left or right of Tables 7 and 8, I decided to shorten the odds and instead pair the most frequently occurring cadence in Riemenschneider (I-PA1) with one that never occurs, de Clercq's Figure-2 cadence, the hypothetical harmonization of the first phrase of "Wie schön leuchtet der Morgenstern" with an HF in the tonic (I-HF1). This drew more positive results; students mostly but not universally identified I-PA1 as sounding more in the manner of Bach than I-HF1.

Being somewhat perplexed, however, that my harmony students didn't fully, or unanimously, appreciate the stylistic (and statistical) difference between the ubiquitous and the non-existent, which a purely style-probability model suggests should be the case, I undertook to explore the matter further. Agreeing with de Clercq's statement that his study may well have "....ignored other important factors in Bach's compositional process" (p. 204) - particularly factors, I suspected, responsible for Bach's compositional style - I decided to include the element of figuration in my exploration.[5][6] My overall (slightly mischievous) intention was to see whether certain figurations might result in students viewing the most ubiquitous cadence, I-PA1, as being less stylistic than the never-occurring cadence referred to above, I-HF 1, and in so doing demonstrate that, with respect to style, figuration holds a trump card. This is not to say that cadence-melody distributions do not contribute to style, merely that if style mastership is a primary goal of harmony classes, an understanding of how figuration interacts with cadence and melodic scale degree might be particularly important.

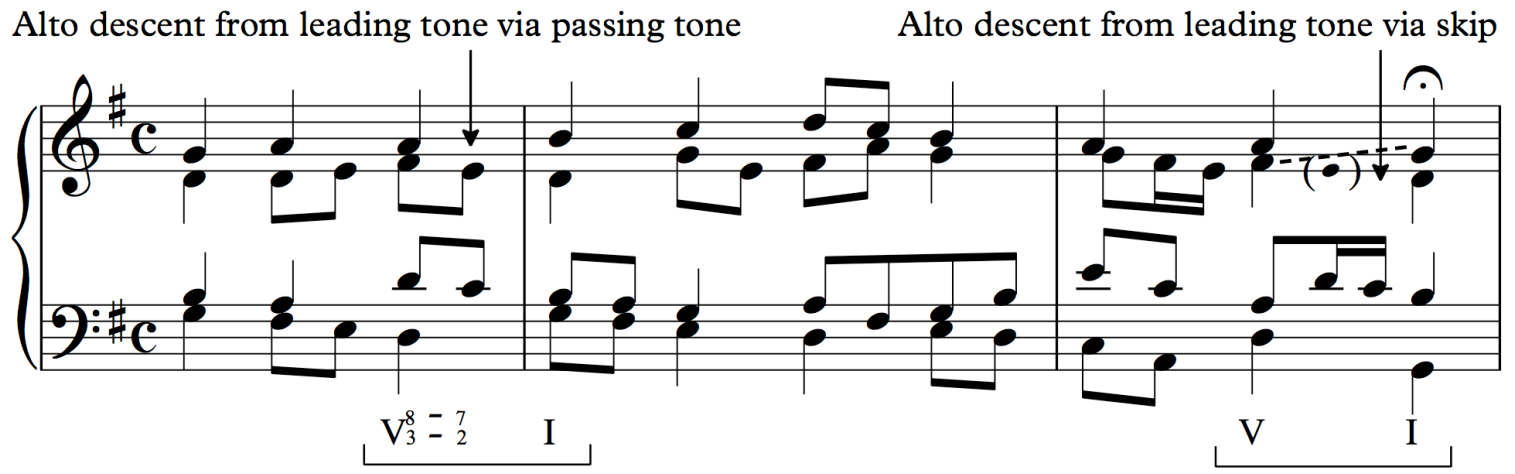

Fig. 1. Third phrase of chorale 124, "Auf, auf, mein Herz, und du mein ganzer Sinn”, containing examples of descent from the leading tone via a passing tone (non-cadence) and skip (cadence).

In line with de Clercq's focus on fermata events, the figuration I had in mind concerned phrase termination. Overriding the tendency of leading tones to rise, sometimes given "rule" status in introductory 
harmony classes, for reasons of chordal balance, at PAs Bach frequently skips down a major third from the leading tone to the fifth of the tonic chord (e.g. final cadences in de Clercq's Figures 6 and 7).[7] Interestingly, Bach almost never fills this descending third with a passing tone at a cadence, despite occasionally doing so at non-cadential points where dominant harmony moves to tonic, as in my Figure 1, the third phrase of chorale 124, "Auf, auf, mein Herz, und du mein ganzer Sinn".[8][9] Various explanations may account for this. A descending passing tone from the leading tone could disrupt the sense of voice exchange at the cadence, indicated by the dotted line in Figure 1, alto to soprano. Or perhaps Bach wanted to avoid parallel fourths at cadences, which, in Figure 1, would have been created between alto and soprano by an alto passing-tone E-natural (shown in brackets); although, elsewhere, Bach is of course not at all shy of parallel fourths. Either way, and for whatever reason, Bach's careful avoidance of stepwise descents from leading-tones at cadences provided an opportunity to create a second never-occurring cadence, to match that of de Clercq's Figure 2: a I-PA1 with a passing tone descending from the leading tone. However, while de Clercq's Figure-2 cadence never occurs because of a unique combination of structural elements - melodic pitch and cadence type - mine was due simply to unconventional figuration. Indeed, the structural elements of my I-PA1 cadence, melodic root and V-I harmony, are the most commonly occurring within Riemenschneider.

To these two cadences, I added a third: I-IA3, also with a passing tone descending from the leading tone. As de Clercq has amply shown, melodic scale degree appears to play a far greater role in determining cadence type than may have previously been thought. My hunch, and the reason for using I-IA3, was that melodic scale degree also plays an important role in admitting certain types of figuration whilst excluding others, which, in turn, might strongly determine stylistic quality. Given that my relatively cursory study yielded at least three instances in which stepwise leading-tone descents were consistently followed by chords with a melodic third (chorales 23, 124 and 163), I reckoned that students might find my I-IA3 cadence to be more stylistic than my I-PA1, despite I-PA1 cadences being more frequent overall; see de Clercq's Table 5. Figure 2 shows the three cadences presented to harmony students, transposed to F major. From left to right, de Clercq's Figure 2 (I-HF1); my I-PA1 with a passing tone descending from the leading tone; my I-IA3 also with a passing tone descending from the leading tone. Readers may have noticed that my two cadences (I-PA1 and I-IA3) are adapted from the dominant-to-tonic progressions in the third phrase of chorale 124, my Figure 1.

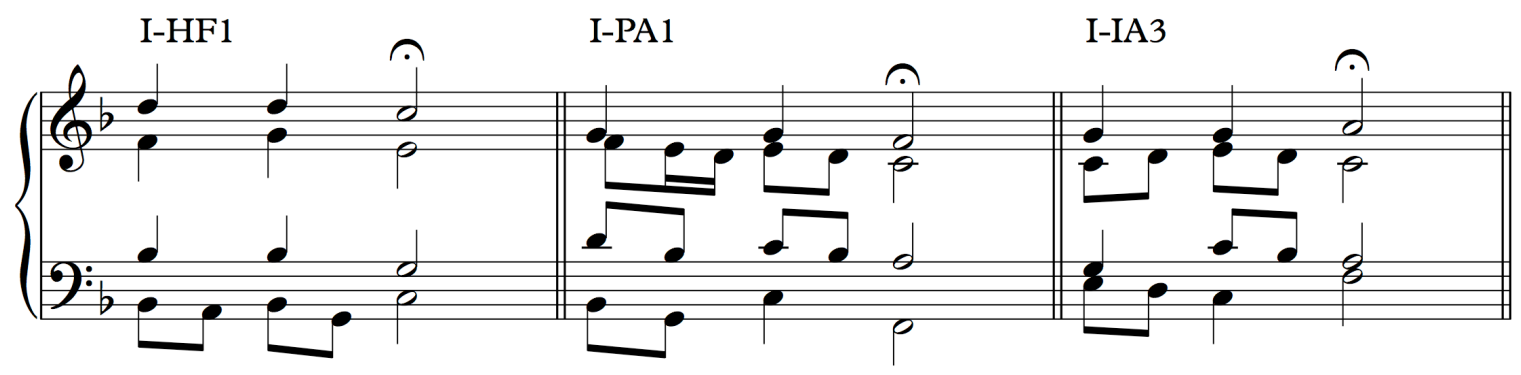

Fig. 2. Three cadences used in the test: I-HF1 (de Clercq, Figure 2); I-PA1 (passing tone descending from leading tone); I-IA3 (passing tone descending from leading tone).

To summarize, the aims of my ad hoc test were twofold. First, an objective was to see whether the addition of a single, unconventional passing tone would result in students viewing the most ubiquitous cadence, I-PA1, as being less stylistic than de Clercq's never-occurring cadence, i.e., I-HF1 vs. I-PA1 from my Figure 2. A second goal was to explore whether students would judge a passing-tone descent from the leading tone as being more stylistic if the following melody note was the third of the chord rather than root, i.e., I-IA3 vs. I-PA1 from my Figure 2. Cadences were played in all possible pairs, with the forced-choice question, "Which of the two is more typical of Bach's chorale style?" Randomly ordered pairs at various transpositions were presented; students voted for the first or second in each pair by entering their choice onto a pre-prepared questionnaire. Votes were summed and a preference-percentage calculated for each cadence. Results were as follows: I-HF1, 50\%; I-PA1, 18\%; I-IA3, 32\%.

At the outset I should state that my rough-and-ready test does not prove or disprove anything-it was performed in a relatively unscientific manner in a classroom, and contained numerous possible confounds that may have skewed the results. However, despite the deficiencies, the outcome is suggestive 
of the following, which I believe to be pertinent to de Clercq's study. First, the considerably higher "authenticity" score for I-HF1 over I-PA1 (50\% to 18\%) indicates that, with respect to style, the simple addition of a passing tone has a far greater effect than frequency of cadence coupled with scale degree: de Clercq's I-HF1 was considered more Bachian than I-PA1. I hasten to add that this finding does not negate de Clercq's sterling work in anyway; however, it should sound a cautionary note to anyone hoping to achieve a pastiche chorale style merely by applying probable cadences given particular scale degrees (which is not what de Clercq is proposing). Of course, it could be argued that I-PA1 received a lower score precisely because of its high probability: we are more sensitive to frequently occurring events (Loui \& Wessel, 2007), and therefore their "violation", through, for example, the inclusion of unconventional figuration, has a more marked effect. Nonetheless, it remains the case that something as simple as a passing tone led to the perception of a never-occurring combination of scale degree and cadence (I-HF1) as being more stylistic than the most frequent combination of scale degree and cadence (I-PA1). And, therefore, demonstrates that harmony instructors, when seeking to achieve stylistic competence among their students, may be well advised to focus the bulk of their efforts on the appropriate use of figuration. Second, the higher score for I-IA3 over I-PA1 (32\% to $18 \%$ ), both of which contain stepwise descents from leading tones, suggests that the influence of melody upon cadence, as found by de Clercq, similarly extends to figuration. Students perceived a stepwise descent from the leading tone to be more stylistic when the following melodic pitch was the third of the chord, rather than the root. On the face of it, and from the examples I have identified, this is borne out in Bach's chorale harmonizations-each instance of a stepwise descent from the leading tone is followed by a melodic third (chorales 23, 124 and 163). Whether this trend is merely coincidental, however, remains to be established, although the students' perceptions certainly point to this phenomenon being more than happenstance. Which is to say, a stepwise descent from a leading tone followed by a melodic third maybe an identifiable style feature, albeit relatively rare, brought about through an interaction of melody and harmonic progression.

In sum, as mentioned earlier, the foregoing suggests that the relationship between style and the probability of a musical event is not straightforward, but nuanced - although neither the I-HF1 or I-PA1 cadences presented to my students are found in Riemenschneider, they received very different levels of approval. Rather, in addition to raw probabilities, style arises through the combination of melodic-pitch, figuration, rhythm and harmonic phenomena, which may occasionally result in relatively infrequent events being highly stylistic. De Clercq has uncovered a particular type of interaction, that of scale degree and cadence. It remains to be seen whether other interactions, perhaps between metre and figuration, lead to equally structured outcomes. As a final example illustrating the way in which a simple probabilitydominated conception of musical style might be questionable, take the opening phrase of chorale 86 "Wie schön leuchtet der Morgenstern", shown in de Clercq's Figure 7. The original hymn melody, written by Philipp Nicolai in 1597, did not contain the thirty-second-note figuration - the addition is Bach's. Thirtysecond-note figures are almost entirely absent in Riemenschneider, yet, oddly perhaps, to me this fleeting moment is quintessential Bach and not at all out of place.[10]

I conclude by briefly discussing de Clercq's study within a broader context of models of music. In his monograph, Music and Probability, David Temperley outlines a formal method by which the predictive capacities of models might be compared and tested (Temperley, 2007, pp. 159-70). Referred to as cross entropy, the method calculates the probability of the musical data as assigned by the model per musical event (e.g. chord, melody note), essentially a measure of the model's "goodness of fit". With respect to evaluating various models, Temperley states, "Each model assigns some probability to the data; the best model is the one that assigns the highest probability" (p. 160). Moreover, Temperley states the best model "...is more likely to describe the generative process that gave rise to the data" (p. 162), but with an important caveat. The ability of a model to explain the cognitive processes involved in a piece's composition is possible only to the extent that the model is comprised of established, plausible psychological and/or cognitive phenomena. Therefore, argues Temperley, if a piece can be modeled effectively using factors known to be psychologically valid, such as scale structure, range and pitch proximity, then we would be justified in positing these as causal to the piece's generation. Which is to say, if a model is based upon bona fide cognitive processes and it models the data well, then these processes are likely to have underpinned the generation of the composition in the first place. Of course, the "best" model, arguably, is one that simply describes each event within a piece, in its entirety. There are two downsides to this, however. First, the model is likely to be highly complex; and second, the descriptive nature of the model is likely to undermine its utility with respect to explicating in a parsimonious manner the cognitive process responsible for the music's creation. 
In light of the above, how might de Clercq's model be evaluated? It is true that it is succinct, as laid out in Tables 7 and 8, and predicts to a pleasing degree Bach's chorale cadences given particular scale degrees. However, with respect to the model possessing explicatory power, i.e., the ability to explain why musical facts are as they are, arguably, as yet it is still in its infancy. So far, de Clercq has produced a series of accurate and insightful observations, for which he is to be congratulated. Also to be appreciated is his decision to craft these observations into a model with pedagogical utility - the flowchart in Figure 18 is potentially very useful to harmony students, particularly those in the early stages still assimilating Bach's chorale style. A challenge now will be to move beyond observation and broaden the model in the direction of cognitive science. This will, one hopes, not only render the model relevant to a wider audience, but also provide that which the model currently lacks: an explanation at a psychological level of Bach's cadential decisions rather than merely their description given certain scale degrees.

Finally, it is worth saying a few words concerning proscriptive approaches to teaching harmony. A danger is that in lackadaisical hands de Clercq's model may be a substitute for hard work, dampening the development of a student's inner ear and aesthetic sense rather than fostering it. Given the heavy workload commonly associated with university life, a temptation can be for students to use any and every educational "tool" at their disposal, irrespective of whether or not it develops a deeper understanding of the subject. This is an unfortunate fact of contemporary higher education that instructors can do little about. However, we do have the discretion to introduce students to aids that may make their study assignments (and lives) easier. The question is not whether the model is good or would lead to better-sounding outcomes-it is, and probably would, certainly in the short term - rather the issue is whether it may remove a vital hurdle, akin to a rite of passage, the surmounting of which is crucial to the development of a student's knowledge of musical style, not to mention their sense of educational achievement. In essence, the issue is between implicit and explicit harmonic knowledge. For generations, musicians have acquired (semi-)implicit style knowledge through intense and prolonged immersion in a particular body of music. The process is gradual, creative, often frustrating, but also punctuated with exhilarating, sustaining flashes of insight. Before replacing that with a model based on probabilistic knowledge, care should be taken to ensure that the valuable element of trial and error is not entirely removed from the picture. So, will I adopt de Clercq's model in my harmony classes? Well, on balance probably yes, but only in so far as it would free up time for students to become more deeply engrossed in the art of figuration, a matter truly central to the appreciation of Bach's chorale style.

\section{NOTES}

[1] Correspondence concerning this article should be addressed to: Matthew Woolhouse, School of the Arts, 1280 Main St. W., Hamilton, Ontario L8S 4M2, CANADA, woolhouse@mcmaster.ca

[2] Presumably the increase in performance is due to the formulaic nature of the descending second at cadences, which led de Clercq to omit the final cadences (dominated by descending melodic seconds) from his analysis.

[3] In fact, ignoring scale degree, the distribution of cadences in de Clercq's Tables 5 and 6 is even more extreme than a Zipf distribution, proportionally falling away to the long tail far more rapidly: PA1, 719; HF5, 322; IA3, 291; DE3, 15; PL5, 12; PH3, 10; SS3, 9.

[4] As an aside, a recommendation in more advanced classes can be to include a verse of the original text with translation, hopefully stimulating students to word-paint, as Bach did, in a way that captures Pietist sentiments of the text.

[5] The idea that figuration is a key component of a composer's style is of course not new. For the significance of figuration in Bach's music, particularly from the Schenkerian perspective of structural elaborations, see Väisälä (2009). For further discussion, see also Dreyfus (1996), Wolff (1990), and Neumann (1983).

[6] Extensive use of melodic and rhythmic figurations can be found in virtually all of Bach's chorale harmonizations. Melodic figuration includes chordal skips, passing tones, neighbor tones, including 
incomplete neighbor tones; rhythmic figurations include suspensions, retardations, anticipations and pedal points (Aldwell \& Schachter, 2003, pp. 371-417).

[7] Within Riemenschneider, chords at fermatas are usually balanced with two roots, a third and a fifth.

[8] As per de Clercq, I am using chorale numbers as found in Riemenschneider.

[9] An example of non-cadential stepwise descent from the leading tone can be found in the second phrase (tenor, measure 3) of chorale 163, "Für Freuden laßt uns springen". For a very rare example of cadential stepwise descent from the leading tone, see the penultimate phrase (alto, measure 10) of chorale 23, "Zeuch ein zu deinen Toren". In this latter case, a simultaneous soprano passing tone (C-natural) partially masks the leading-tone descent in the alto.

[10] Given the parameters of the analysis, de Clercq's decision to ignore the thirty-second-note figure is entirely justified. However, from a style perspective, the choice to nullify Bach's contribution and move back to Nicolai's original melody could be viewed as an instance of throwing the musical baby out with the bathwater.

\section{REFERENCES}

Aldwell, E., \& Schachter, C. (2010). Harmony and voice leading (4th ed.). Cengage Learning.

Bach, J. S. (Ed.: A. Riemenschneider). (1941). 371 Harmonized chorales and 96 chorale melodies. Milwaukee, WI: G. Schirmer.

Conklin, D. (2006). Melodic analysis with segment classes. Machine Learning, 65(2-3), 349-360.

Creel, S. C., Newport, E. L., \& Aslin, R. N. (2004). Distant melodies: Statistical learning of nonadjacent dependencies in tone sequences. Journal of Experimental Psychology: Learning, Memory, and Cognition, 30(5), 1119.

De Clercq, T. (2015). A model for scale-degree reinterpretation: Melodic structure, modulation, and cadence choice in the chorale harmonizations of J. S. Bach. Empirical Musicology Review, 10, 188-206.

De Clercq, T., \& Temperley, D. (2011). A corpus analysis of rock harmony. Popular Music, 30(1), 47-70.

Dreyfus, L. (1996). Bach and the patterns of invention. Harvard University Press.

Dubnov, S., Assayag, G., Lartillot, O., \& Bejerano, G. (2003). Using machine-learning methods for musical style modeling. Computer, 36(10), 73-80.

Krumhansl, C. L. (1995). Music psychology and music theory: Problems and prospects. Music Theory Spectrum, 17(1), 53-80.

Kuhn, G., \& Dienes, Z. (2005). Implicit learning of nonlocal musical rules: Implicitly learning more than chunks. Journal of Experimental Psychology: Learning, Memory, and Cognition, 31(6), 1417-1432.

Lester, J. (1999). Bach's works for solo violin: style, structure, performance. Oxford: Oxford University Press.

Loui, P., Wessel, D. L., \& Kam, C. L. H. (2010). Humans rapidly learn grammatical structure in a new musical scale. Music Perception, 27(5), 377-388.

Loui, P., Wu, E. H., Wessel, D. L., \& Knight, R. T. (2009). A generalized mechanism for perception of pitch patterns. The Journal of Neuroscience, 29(2), 454-459. 
Loui, P., \& Wessel, D. (2007). Harmonic expectation and affect in western music: Effects of attention and training. Perception \& Psychophysics, 69(7), 1084-1092.

McHose, A. (1947). The contrapuntal harmonic technique of the 18th century. New York: Meredith Publishing.

Meyer, L. B. (1957). Meaning in music and information theory. Journal of Aesthetics and Art Criticism, 15, 412-424.

Meyer, L. B. (1956). Emotion and meaning in music. Chicago: University of Chicago Press.

Narmour, E. (1990). The analysis and cognition of basic melodic structures: The implication-realization model. Chicago: University of Chicago Press.

Neumann, F. (1983). Ornamentation in baroque and post-baroque music: With special emphasis on JS Bach. New Jersey: Princeton University Press.

Parncutt, R. (1997) A model of the perceptual root(s) of a chord accounting for voicing and prevailing tonality. In M. Leman (Ed.), Music, Gestalt and Computing: Studies in Cognitive and Systematic Musicology (pp. 181-199). Berlin: Springer.

Pearce, M. T., Ruiz, M. H., Kapasi, S., Wiggins, G. A., \& Bhattacharya, J. (2010). Unsupervised statistical learning underpins computational, behavioural, and neural manifestations of musical expectation. NeuroImage, 50(1), 302-313.

Pearce, M., \& Wiggins, G. (2004). Improved methods for statistical modelling of monophonic music. Journal of New Music Research, 33, 367-385.

Pearce, M. T., \& Wiggins, G. A. (2006). Expectation in melody: The influence of context and learning. Music Perception, 23, 377-405.

Piston, W. (1978). Harmony (4th ed.). New York: Norton. (Originally published, 1941.)

Rohrmeier, M., \& Cross, I. (2008). Statistical properties of tonal harmony in Bach's chorales. In Proceedings of the 10th International Conference on Music Perception and Cognition (pp. 619-627). Hokkaido University Sapporo, Japan.

Saffran, J. R., Johnson, E. K., Aslin, R. N., \& Newport, E. L. (1999). Statistical learning of tone sequences by human infants and adults. Cognition, 70(1), 27-52.

Temperley, D. (2007). Music and probability. The MIT Press.

Väisälä, O. (2009). Bach's inventions: figuration, register, structure, and the "Clear Way to Develop Inventions Properly". Music Theory Spectrum, 31(1), 101-152.

Wolff, K. (1990). Masters of the keyboard: Individual style elements in the piano music of Bach, Haydn, Mozart, Beethoven, Schubert, Chopin, and Brahms (Vol. 567). Bloomington, IN: Indiana University Press.

Zanette, D. H. (2006). Zipf's Law and the creation of musical context. Musicae Scientiae, 10(1), 3-18. 